\title{
Future upgrades of ALICE for Run 4
}

\section{Filip Krizek ${ }^{a, *}$ for the ALICE Collaboration}

${ }^{a}$ Nuclear Physics Institute of CAS,

Husinec-Rez 130, Czechia

E-mail: krizek@ujf.cas.cz

The ALICE Collaboration is preparing two upgrades for installation during LHC Long Shutdown 3. The first project is the replacement of the inner barrel of the Inner Tracking System (ITS) detector with cylindrical layers based on large-scale, wafer-sized, bent, monolithic active pixel sensors. This technology will provide a detector with an unprecedentedly low material budget and will allow unparalleled vertexing performance. The second project will add the novel concept of a Forward Calorimeter (FoCal), consisting of a Si-W electromagnetic calorimeter, with pad and pixel readout, and a conventional metal-scintillator hadronic calorimeter. The design of the electromagnetic calorimeter aims for high spatial resolution for the separation of nearby photon showers. This article presents the R\&D programs of both projects and discusses the prospects for physics.

\footnotetext{
*** The European Physical Society Conference on High Energy Physics (EPS-HEP2021), *** *** 26-30 July $2021 * * *$

*** Online conference, jointly organized by Universität Hamburg and the research center DESY ***
}

\footnotetext{
*Speaker
} 


\section{Introduction}

The Run 3 and 4 data taking periods of the experiments at the CERN Large Hadron Collider are scheduled for the years 2022-2024 and 2027-2030, respectively. The ALICE collaboration has proposed a physics program for these two periods that largely focuses on precise measurements of low- $p_{\mathrm{T}}$ open heavy-flavour hadrons and quarkonia, low-mass dielectrons, and the production of light nuclei, antinuclei and hypernuclei [1]. This physics program calls for major detector upgrades, in particular continuous data readout, to collect a hundred times more data with respect to Run 1 and Run 2. The upgrades during the Long Shutdown 2 were the replacement of the ALICE silicon tracker by a new Inner Tracking System (ITS) detector [2], the installation of the new Muon Forward Telescope and Fast Interaction Trigger detectors [3, 4], the upgrade of the Time Projection Chamber readout [5], and finally also the development of the new Online-Offline computing system [6] that will be used for data processing during the data taking. This proceedings focuses on two additional upgrades, which are foreseen for Run 4 . The first upgrade aims to further significantly reduce the material budget of the inner barrel of the ITS and the second upgrade will instrument the forward pseudorapidity of ALICE with a new calorimeter.

\section{Inner barrel of the ITS in Run 4}

For Run 3, the ITS detector will consist of seven cylindrical layers of monolithic active pixel sensors called ALPIDE [7]. The inner barrel is formed by the first three layers, where the radial distance of the first layer from the beam is just $22 \mathrm{~mm}$. The material budget of each of the three layers is $0.35 \% \mathrm{X}_{0}$ only. The ALPIDE chip has dimensions $3 \times 1.5 \mathrm{~cm}^{2}$ and in the inner barrel it is thinned down to $50 \mu \mathrm{m}$. The ALPIDE sensor has binary readout with zero suppression, which allows average power density below $40 \mathrm{~mW} \mathrm{~cm}^{-2}$. Yet, the composition of the inner barrel material budget is to a large extent formed by passive components such as water cooling, carbon and kapton support structures, and aluminum wires, see Fig. 1. The silicon accounts for $15 \%$ of the material budget only. The new design of the ITS inner barrel for Run 4 therefore aims to avoid the passive material keeping essentially just the silicon layer [8]. Such a detector would achieve an unprecedentedly low material budget of about $0.05 \% \mathrm{X}_{0}$ per layer. The construction of the detector would require (i) reducing the power consumption below $20 \mathrm{~mW} \mathrm{~cm}^{-2}$, in order that the sensor can be cooled by airflow, (ii) to integrate power and data buses on the chip, and (iii) to rely on the stiffness of large size, bent silicon wafers. Such a low value for the power consumption can be achieved by moving the sensor periphery, including the serial link, to the edge of the wafer-sized chip and by the use of $65 \mathrm{~nm}$ CMOS technology.

The new design for the ITS inner barrel for Run 4 is shown in Fig. 1. It will have also three layers of monolithic active pixel sensors. These sensors will be $28 \mathrm{~cm}$ long and they will be thinned down to about $40 \mu \mathrm{m}$ such that they can be easily bent. The production of sensors of this size relies on $2 \mathrm{D}$ stitching technology on $300 \mathrm{~mm}$ wafers.

The new design makes it possible to move the innermost layer from $22 \mathrm{~mm}$ to $18 \mathrm{~mm}$ from the beam. Mechanical support of the silicon layers will be provided by low-material-budget carbonfoam spacers. 

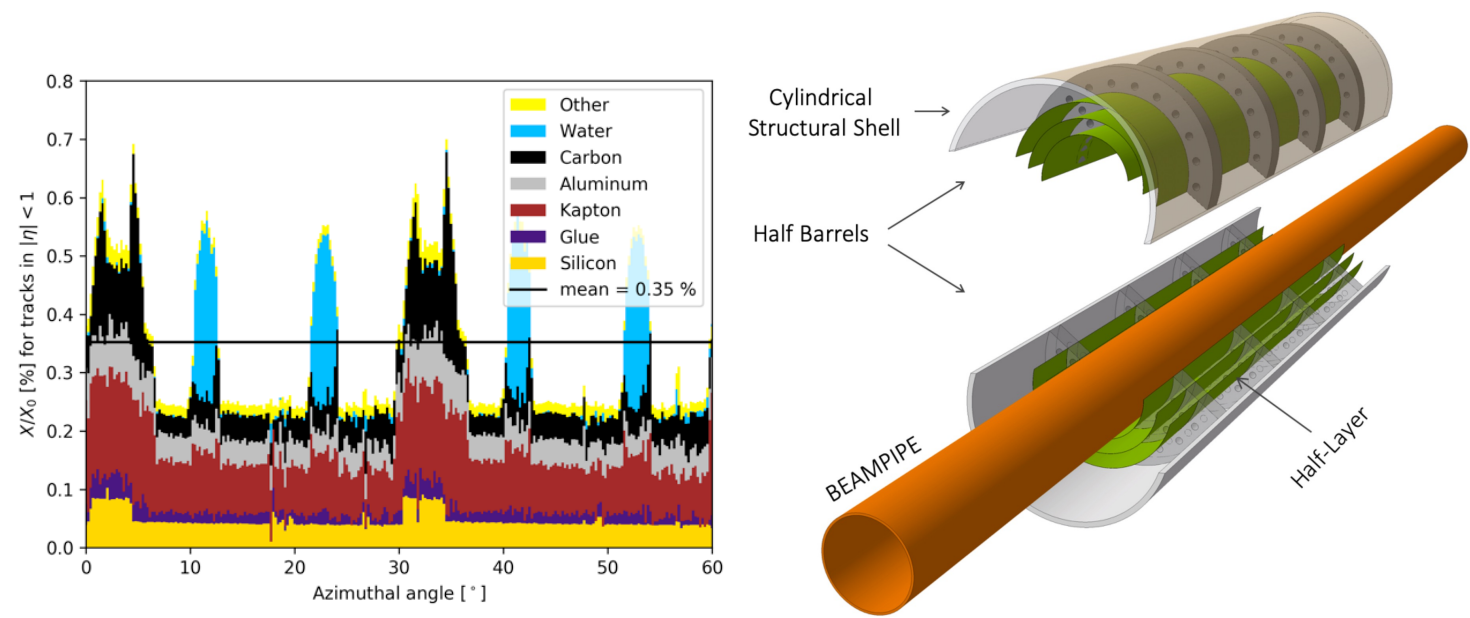

Figure 1: Left: Material budget of a single ITS inner barrel layer in Run 3. The thickness of the silicon is $\approx 0.05 \% \mathrm{X}_{0}$. Right: Proposed design for the inner barrel of the ITS in Run 4.

The expected gain in performance with the new detector in Run 4 with respect to Run 3 is shown in Fig. 2. The new detector design improves the pointing resolution of tracks in the plane transverse to the beam by about a factor of 2 and in addition, it enhances the reconstruction efficiency for low momentum particles. The expected impact on heavy-flavour hadron reconstruction can be illustrated for the case of the $\Lambda_{c}$ particle where the significance of the invariant mass peak should increase by a factor 4 [8].
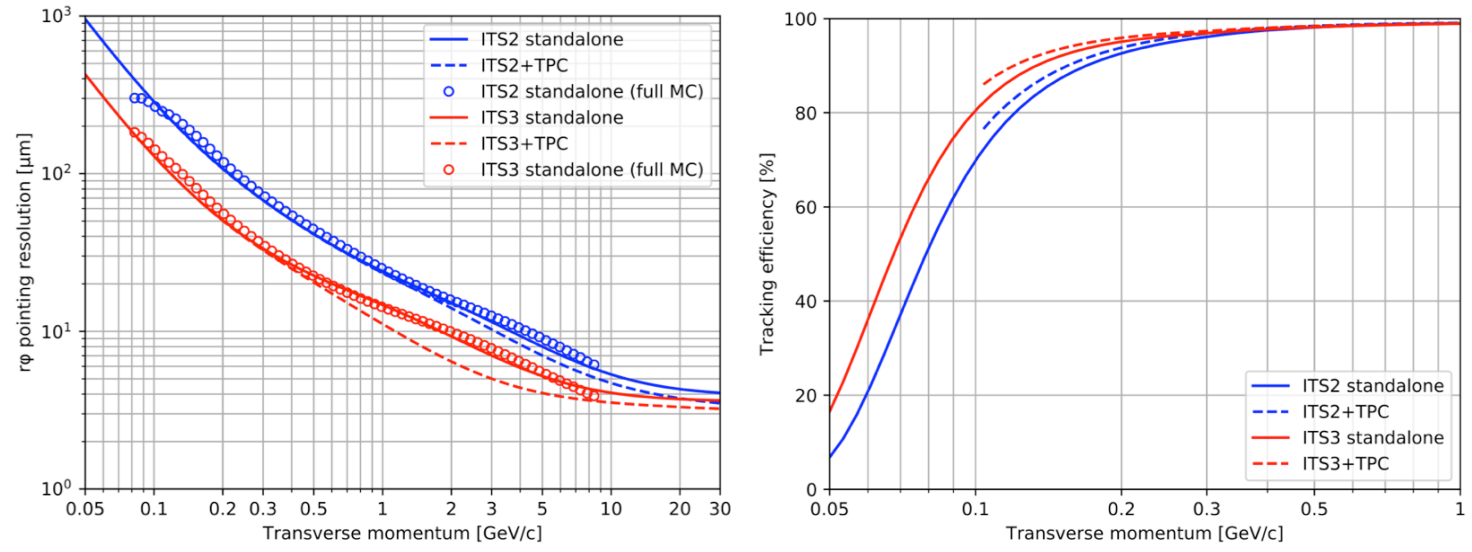

Figure 2: Comparison of the tracking performance in Run 3 (blue colour) and Run 4 (red colour). Left: Pointing resolution of tracks in the plane transverse to the beam. Right: Tracking efficiency. Taken from [8].

Current R\&D studies focus on the construction of a full size mechanical prototype and the characterization of bent sensor properties. It has been shown that large sheets of silicon can be bent to the desired radii using a mechanical mandrel and that bent ALPIDE sensors retain their performance. The hit efficiency was tested in the first beam test where a bent ALPIDE sensor was irradiated using a $5 \mathrm{GeV}$ electron beam [9]. Future test beams will be carried out with the so called $\mu \mathrm{ITS} 3$, formed by 3 layers of bent ALPIDE sensors having the same radii as the future inner barrel. 


\section{Forward calorimeter}

The Forward Calorimeter (FoCal) [10] will cover the forward pseudorapidity of the ALICE detector in the range $3.4<\eta<5.8$. The detector will focus on measurements of isolated photons and $\pi^{0} \mathrm{~s}$ in the region of small Bjorken $x$ and small four-momentum transfer, $Q$, where the onset of gluon saturation in lead nuclei is expected to emerge, see Fig. 3. One of the key goals of the FoCal is to provide new unique data that would allow us to constrain the current nuclear parton distribution functions in the region $10^{-6}<x<10^{-3}$, where they suffer from large uncertainties [11]. Moreover, the FoCal will also be used to explore jet quenching at forward rapidity and it will significantly extend the baseline for measurements of long-range flow-like correlations in ALICE.
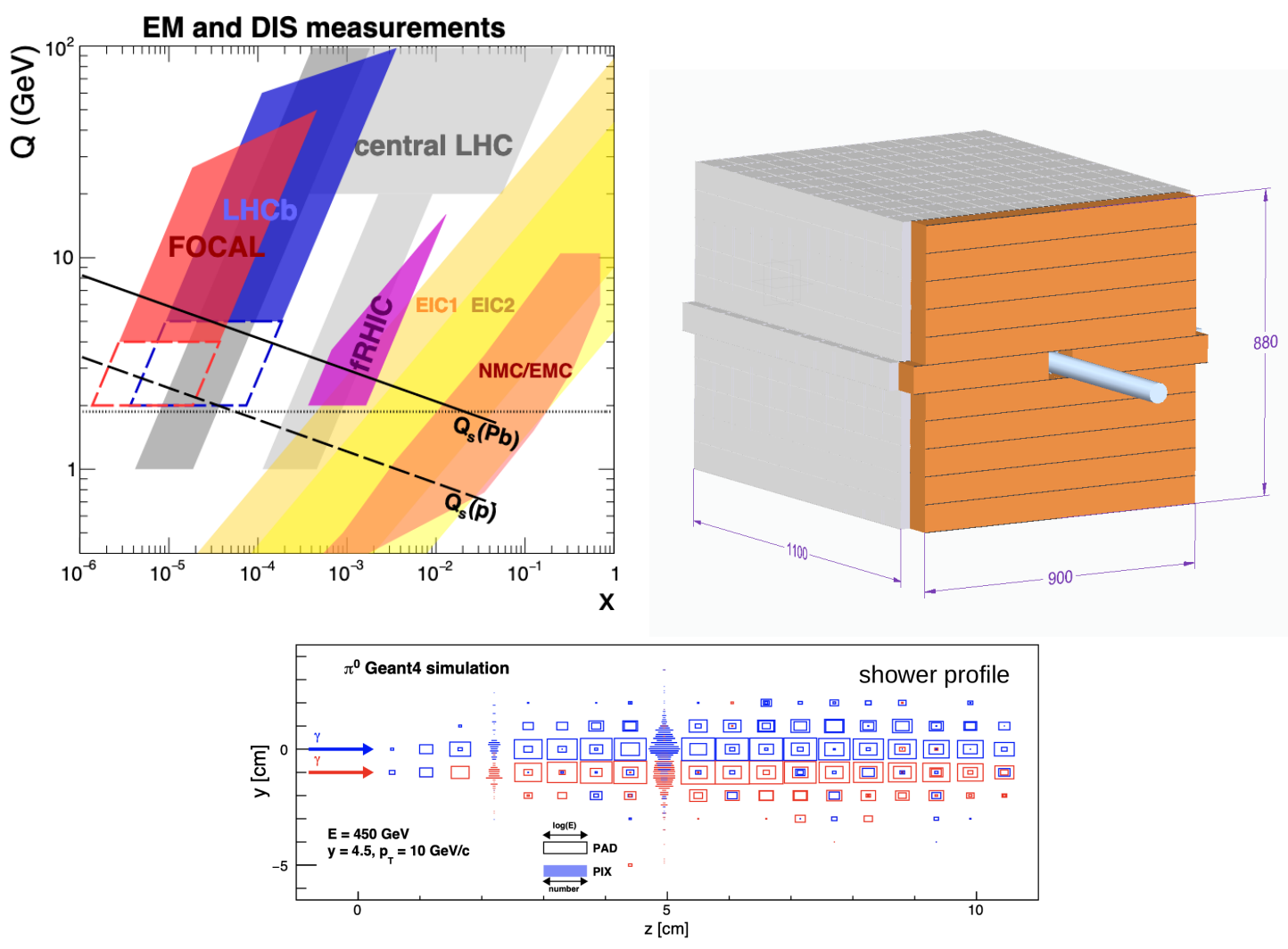

Figure 3: Top left: Approximate $(x, Q)$ coverage for measurement of deep inelastic scattering in various experiments. The saturation scale in lead nucleus is denoted as $Q_{\mathrm{S}}(\mathrm{Pb})$. Top right: FoCal detector centered around the LHC beam pipe. The electromagnetic calorimeter is depicted in brown and the hadronic calorimeter in grey colour. The quoted dimensions are in millimeters. Bottom: Simulated spatial separation of two electromagnetic showers in Focal. The two initial photons were displaced by $1 \mathrm{~cm}$ in the transverse plane. Size of the colour boxes shows the charge integrated in silicon pads. It can be seen that the high-granularity layers provide the crucial power to identify such events. Taken from [10].

The FoCal design is presented in Fig. 3. The detector will be placed $7 \mathrm{~m}$ from the interaction point and consist of an electromagnetic and a hadronic calorimeter. The hadronic calorimeter will be a conventional, hadronic sampling calorimeter of 6 interaction lengths. The electromagnetic calorimeter will be a $20 \mathrm{X}_{0}$ thick silicon-tungsten sampling calorimeter with 18 layers of tungsten 
absorbers and silicon sensors. Low-granularity silicon sensors (pad size $1 \mathrm{~cm}^{2}$ ) with fast integration time for charge collection will be used in 16 out of the 18 layers. The 2 remaining layers shall be equipped with high-granularity monolithic active pixel sensors based on the ALPIDE sensor technology with binary readout and slower integration time of about $5 \mu$ s. The high-granularity layers are going to be placed at the positions, where an electromagnetic shower reaches its maximum (layers 5 and 10). The main role of these layers is to identify overlapping showers, see the bottom panel in Fig. 3. The smallest transverse separation when two showers can be still distinguished is $2 \mathrm{~mm}$. Direct photon candidates will be selected by means of an isolation criterion by imposing an upper limit on the energy deposited in a cone centered around the shower. The purity of the direct photons will be further enhanced by rejecting pairs of clusters having an invariant mass close to that of the $\pi^{0}$. This enhances the direct photon fraction by about a factor 20 and yields a purity of about $50 \%$ for photons with transverse momentum larger than $7 \mathrm{GeV} / c$ in pp collisions.

Figure 4 shows two calorimeter prototypes which were constructed during the FoCal R\&D. The first prototype, called EPICAL-2, is a small fully digital silicon-tungsten calorimeter with high granularity, which is fully based on the ALPIDE sensors. The calorimeter has 24 layers consisting of a $3 \mathrm{~mm}$ thick tungsten absorber followed by 2 ALPIDE sensors. The transverse cross section of the EPICAL-2 is $3 \times 3 \mathrm{~cm}^{2}$. The second calorimeter prototype is called Mini-FoCal. This detector has 20 layers each containing a tungsten absorber and three low-granularity silicon sensor planes with $8 \times 8$ matrix formed by $1 \mathrm{~cm}^{2}$ silicon pads. The calorimeter prototypes exhibit good linearity and have compatible energy resolution, $\sigma_{E} / E$. The resolutions obained were $0.19 / \sqrt{E} \oplus 0.01$ in the case of the EPICAL-2 and 0.17/ $\sqrt{E} \oplus 0.019$ in the case of the Mini-FoCal. In 2018, the Mini-FoCal was installed in the ALICE cavern to verify the background in situ.

In summary, the ALICE upgrade projects for Run 4 advance on R\&D. The ITS inner barrel project has carried out the first test beam characterization of bent monolithic active pixel sensors which demonstrated that the bending does not deteriorate properties of ALPIDE sensors. Furthermore, it has been also shown that one is able to build a full scale mechanical model. These achievements are important milestones on the way to the new ultralight ITS inner barrel, which will be based on the novel technology of large scale, bent monolithic active pixel sensors. At the same time, the FoCal project prepared two detector prototypes. Their performance was tested during several test beams and it was found to be compatible with the requirents that are needed to achieve the physics goals of the project.

\section{Acknowledgments}

This work was supported by the Ministry of Education, Youth and Sports of the Czech Republic projects LM2018104 and LTT17018.

\section{References}

[1] ALICE Collaboration, Upgrade of the ALICE Experiment: Letter of Intent, J. Phys. G 41 (2014), 087001.

[2] ALICE Collaboration, Technical Design Report for the Upgrade of the ALICE Inner Tracking System, CERN-LHCC-2013-024. 


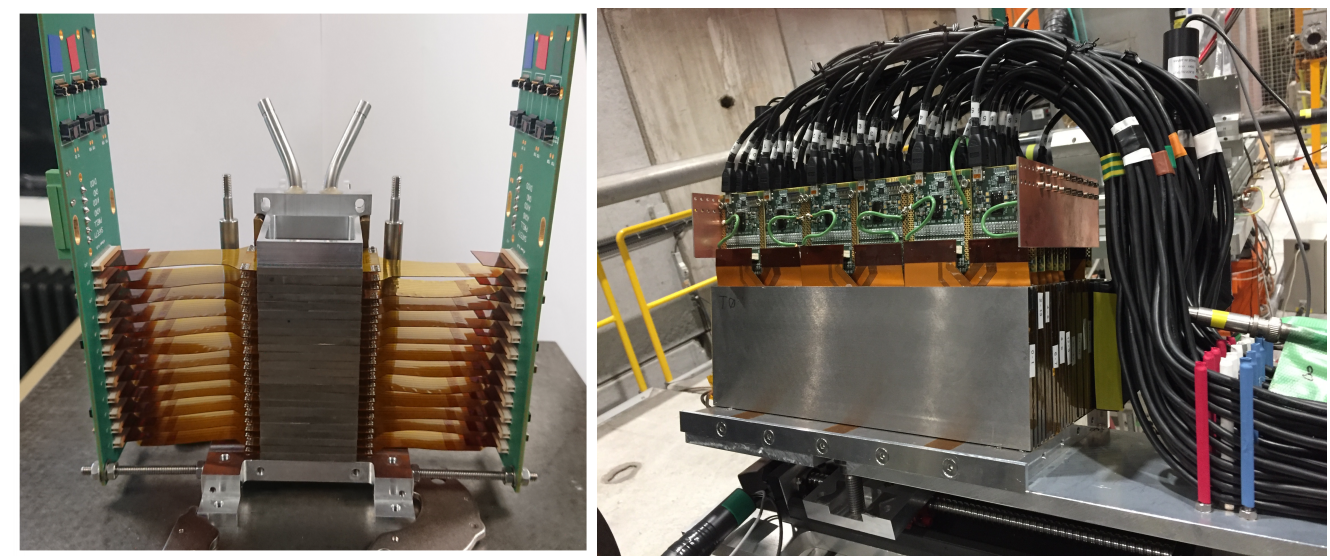

Figure 4: Left: Small, fully digital silicon pixel calorimeter prototype EPICAL-2. Right: Mini-FoCal calorimeter prototype with low granularity silicon planes. Taken from [10].

[3] ALICE Collaboration, Technical Design Reportfor the Muon Forward Tracker, CERN-LHCC2015-001.

[4] ALICE Collaboration, Upgrade of the ALICE Readout \& Trigger System, CERN-LHCC-2013019.

[5] ALICE Collaboration, Technical Design Report for the Upgrade of the ALICE Time Projection Chamber, CERN-LHCC-2013-020.

[6] ALICE Collaboration, Upgrade of the Online-Offline computing system, CERN-LHCC-2015006.

[7] G. Aglieri Rinella et al. (ALICE Collaboration), The ALPIDE pixel sensor chip for the upgrade of the ALICE Inner Tracking System,Nucl. Instrum. Meth. A 845 (2017), 583-587.

[8] ALICE Collaboration, Expression of Interest for an ALICE ITS Upgrade in LS3, ALICEPUBLIC-2018-013.

[9] G. Aglieri Rinella et al. (ALICE ITS Project), First demonstration of in-beam performance of bent Monolithic Active Pixel Sensors, arXiv:2105.13000 [physics.ins-det] .

[10] ALICE Collaboration, Letter of Intent: A Forward Calorimeter (FoCal) in the ALICE experiment, CERN-LHCC-2020-009.

[11] R.A.Khalek et al., nNNPDF2.0: quark flavor separation in nuclei from LHC data, JHEP 09 (2020) 183. 